\title{
A Meta-Analysis of the Global Distribution Pattern of Condensed Tannins in Tree Leaves
}

\author{
Masayuki Ushio ${ }^{1}$ and Jonathan M. Adams ${ }^{*}, 2$ \\ ${ }^{I}$ Center for Ecological Research, Kyoto University, 2-509-3 Hirano, Otsu, Shiga, 520-2113, Japan \\ ${ }^{2}$ Department of Biological Sciences, Seoul National University, Gwanak-Ro, Seoul 151, Republic of Korea
}

\begin{abstract}
Condensed tannins (CT's) are a dominant class of plant secondary metabolites that play important roles in regulating ecosystem processes such as herbivory, decomposition, and nutrient cycling. However, the factors shaping the global distribution pattern of the abundance of CT's in tree leaves are still poorly understood. The purpose of this study was to assess the global distribution pattern of $\mathrm{CT}$ abundance in tree leaves in order to examine the effects of climatic parameters (air temperature and precipitation) and latitude as well as the effects of methodologies used for the measurement of $\mathrm{CT}$ concentration (extract solutions and standard substance). Through a database search, we collected 805 individual values of CT concentration in tree leaves from 72 study sites, which were reported in 26 individual studies as well as mean annual temperature, annual precipitation, and latitude. We also collected the information of extract solution and standard substance if available. We found that mean annual temperature positively correlated with the foliar CT abundance. On the other hand, annual precipitation and latitude did not have significant influences on CT abundance. We did not find significant effects of the extract solution and standard substance on the CT abundance, neither. A higher allocation of carbon to CT's may be advantageous in warmer regions, where the potential herbivore pressure may be relatively higher than that in cooler regions; however, this hypothesis could not be directly tested in this study. Our finding that foliar CT abundance is significantly associated with mean annual temperature supports the 'classical' picture of the stronger plant defenses in warmer climates. A comprehensive empirical comparison using standardized methods is still required to improve our understanding of $\mathrm{CT}$ abundance in tree leaves and the mechanism of ecosystem processes.
\end{abstract}

Keywords: Acid-butanol method, annual precipitation, condensed tannins, global pattern, mean annual temperature, metaanalysis, latitude.

\section{INTRODUCTION}

Condensed tannins (CT's) are a dominant class of plant secondary metabolites that play important roles in regulating ecosystem processes such as herbivory, decomposition, and nutrient cycling. For instance, earlier studies indicated that a higher concentration of foliar CT's reduces the damage from herbivores (Forkner et al. 2004; Feeny 1970). Additionally, CT's can retard the nitrogen mineralization rate by forming a recalcitrant complex with proteins, which in turn affects nitrogen acquisition in plants (Northup et al. 1995; Northup et al. 1998; Ushio et al. 2009). The effects of CT's on the processes of herbivory and decomposition are attributable to the biochemical properties of CT's. CT molecules have many hydroxyl functional groups, which can form hydrogen bonds with the carbonyl functional groups present in proteins (Hagerman and Butler 1981). This protein-precipitation ability of CT's inhibits the activities of proteolytic enzymes and retards the rate of nitrogen mineralization and/or digestion processes in the herbivore guts (Kraus et al. 2003).

*Address correspondence to this author at the Department of Biological Sciences, Seoul National University, Gwanak-Ro, Seoul 151, Republic of Korea; Tel: +82 2880 4404; Fax: +82 2872 1993;

E-mail: jonadams@snu.ac.kr
Furthermore, the antioxidative activity of CT's also retard the degradation processes by protecting substrates from oxidative damage during digestion (Hagerman et al. 1998). These retardations consequently regulate the rate of herbivory and nutrient cycling. Therefore, the measurement of foliar CT abundance and the factors that influence this value would provide insights into the understanding of ecosystem processes.

On the one hand, at a local spatial scale, the foliar CT concentration varies widely depending on plant species, developmental stage of plants, and soil nutrient availability. Earlier studies indicated that plants growing on infertile soil (i.e., in soils with a low concentration of available nutrients and/or acidic soil) contained a higher concentration of foliar CT's than plants growing on fertile soil. For example, McKey et al. (1978) investigated the CT abundance in tree leaves in two African forests with contrasting soil nutrient availability. They found that CT abundance in tree leaf tissues was higher in the forest with nutrient-poor soil than in the forest with nutrient-rich soil. Theoretical and conceptual studies have proposed explanations and justification for this distribution pattern (e.g., Bryant et al. 1983; Coley et al. 1985), and the idea that plants growing on nutrient-poor soils generally contain a larger amount of foliar CT's than those growing on nutrient-rich soils has been commonly accepted by ecologists. 
On the other hand, at the regional scale, researchers predicted that $\mathrm{CT}$ abundance increased with increasing air temperature or decreasing latitude. This prediction is based on the following two points: First, damage by herbivores can be reduced in plants with a higher concentration of foliar CT's (Forkner et al. 2004; Feeny 1970). Second, the herbivore pressure could increase with increasing air temperature or decreasing latitude (Coley and Aide 1991). Therefore, a higher concentration of foliar CT's could be an adaptive trait of plants growing under a high temperature condition, where potential herbivore pressure is high. A few other studies have investigated the latitudinal pattern of the foliar CT abundance. Coley \& Aide (1991) and Coley \& Barone (1996) also suggested that both plant allocation to defensive substances and herbivore pressure could be higher in tropical regions than in temperate regions. Higher air temperature could enhance the herbivore activity, which could result in the higher herbivore pressure in tropical regions. These studies suggest that $\mathrm{CT}$ abundance increases with increasing temperature even at a global scale.

However, due to the lack of global-scale assessment studies, the factors shaping the global distribution pattern of CT abundance are still poorly understood. The lack of such studies appears to be due to the following reasons: The first and most obvious reason is that collecting leaf samples on a global scale would be an enormously time- and effort-consuming enterprise. This problem can be solved by collecting values of foliar $\mathrm{CT}$ abundance for different regions from the published literature. The second reason is that although a considerable amount of data on the foliar CT abundance has been collected, variations in sample processing conditions and measurement methodology prevents the compilation and analyses of the data. For example, the acid-butanol assay (Porter et al. 1986) is the most commonly used technique for the estimation of CT concentration, but there are other alternative methods. Standard tannins that are used to develop standard curves for calibration also vary. Furthermore, different extract solutions are used for tannin extraction from leaf samples. These variations in the extraction methodologies are suggested to influence the quantification of CT's in leaf samples (Cork and Krockenberger 1991). Thus, the abovementioned factors should be given significant consideration while studying the global distribution pattern of CT abundance.

The purpose of this study was to illustrate the global distribution pattern of CT abundance and examine the climatic factors (air temperature and precipitation) and latitude influencing CT abundance at the global scale. We especially focused on CT's in tree leaves, because trees are the major component of global terrestrial vegetation, and because focusing on one life form (i.e. trees) enables us to compare different studies more easily. To this end, we collected the values of $\mathrm{CT}$ concentration from the published literature. Our specific hypothesis was that CT concentration increases with increasing air temperature, and therefore, CT's in leaves should be found at higher concentrations in low-latitude regions. As our secondary objective, we briefly explore the effects of methodologies (i.e., extract solution and standard substance) on CT concentration.

\section{METHODS}

\section{Literature Search}

Our database was compiled by conducting keyword searches using SCOPUS (Elsevier) (Norris and Oppenheim 2007). Combinations of the following key words were used for the database search: "condensed tannins," "herbivory," "decomposition," and "leaves". With this search, more than 100 publications were retrieved. Among these 100 studies, each study to be included in the analysis had to satisfy the following criteria: (1) The CT concentration was evaluated by acid-butanol assay (Porter et al. 1986). (2) The CT concentration was measured in mature leaves of mature trees growing under natural conditions and in growing season. If the CT concentrations were measured for leaves grown under experimental conditions, the study was excluded from our dataset. The acid-butanol assay was chosen because the majority of studies which examined CT concentration used this method. Our data set contains both deciduous trees and evergreen trees.

We collected the following three datasets to accomplish our study objectives. (1) The data on the CT concentration was obtained to determine the global distribution pattern of CT's. The CT concentrations were collected from figures and/or tables in the previous literatures. If only the means of the CT concentrations were reported in the literature, we collected the mean CT concentrations. (2) The data on mean annual temperature, annual precipitation, and latitude were collected to examine the relationships between climatic dataset and the CT concentration. When climatic data and/or latitude data were not available from the publication, we contacted authors, or obtained the data from websites (climatic data, International Water Management Institute [http://wcatlas.iwmi.org/default.asp]; latitude data, Google earth [Google, USA]). (3) The data on chemicals used as extracting solutions for acid-butanol assay and the data on standard substances used for calibrating the CT concentration were collected to examine the dependence of methodologies on the quantification of CT concentration. These methodologies have been suggested to influence the estimation of CT concentrations. (Cork and Krockenberger 1991). By performing the database search as described above, we obtained 805 individual values of CT concentration from 72 study sites reported by 26 individual studies (Table $\mathbf{S 1}$ and Fig. 1).

\section{Statistical Analysis}

We explored the best model to describe the relationship between CT abundance and mean annual temperature, annual precipitation, and latitude. Because the relationship between CT abundance and the variables seems not to be linear (i.e. we did not have a priori hypothesis about the shapes of functions of the variable effects), and because variation of $\mathrm{CT}$ abundance depends on a study site, additive model or additive mixed model were chosen for our current data set. We set seven possible formulae that could explain the relationship between CT abundance and the parameters, those were (1) CT $\sim \mathrm{s}$ (mean annual temperature), (2) CT $\mathrm{s}$ (annual precipitation), (3) $\mathrm{CT} \sim \mathrm{s}$ (absolute latitude), (4) CT 


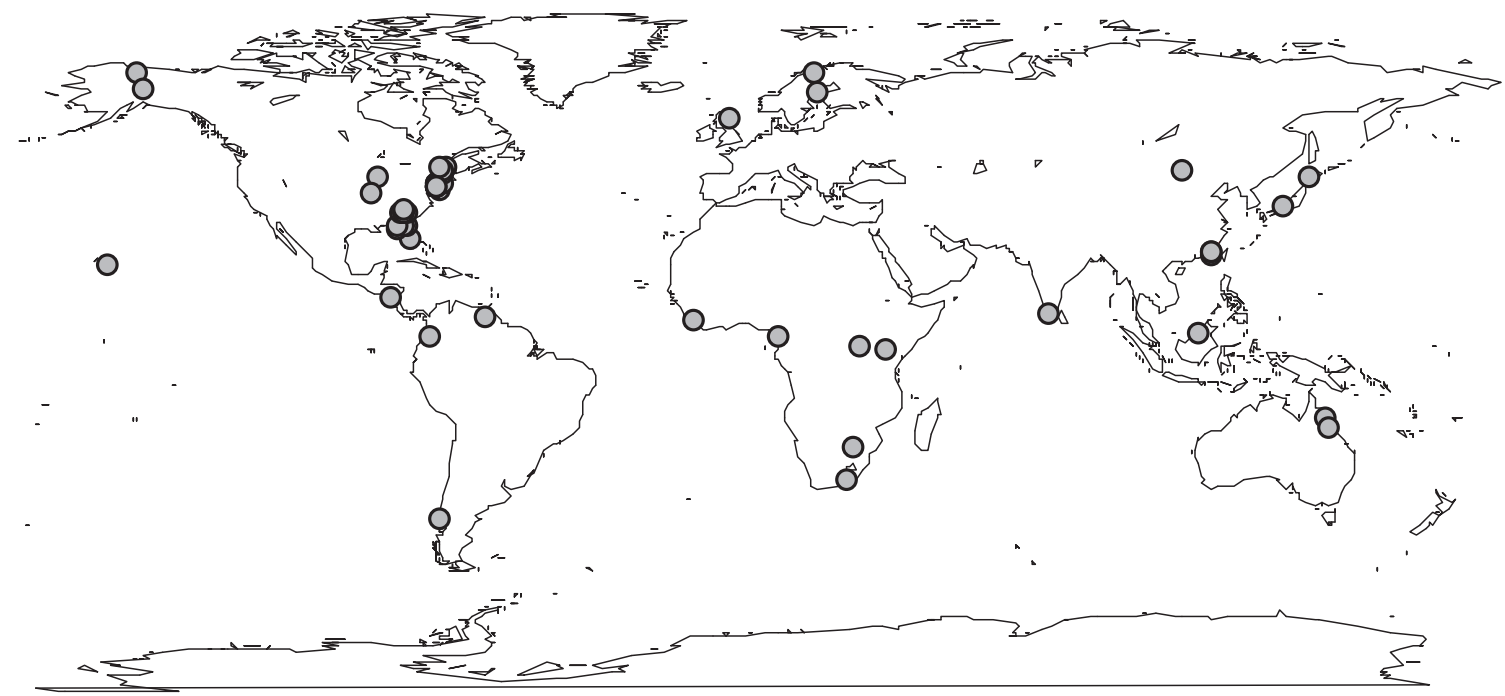

Fig. (1). Study sites included in our dataset. Each gray point indicates a study site from where the data was collected.

$\sim \mathrm{s}($ mean annual temperature $)+\mathrm{s}($ annual precipitation $),(5)$ $\mathrm{CT} \sim \mathrm{s}($ mean annual temperature $)+\mathrm{s}($ absolute latitude $),(6)$ $\mathrm{CT} \sim \mathrm{s}$ (annual precipitation) $+\mathrm{s}($ absolute latitude $),(7) \mathrm{CT} \sim$ $\mathrm{s}$ (mean annual temperature) $+\mathrm{s}$ (annual precipitation) + $\mathrm{s}$ (absolute latitude). Smoothing curve is applied for a term enclosed by $s()$. Interaction terms were not considered because we did not have a specific hypothesis that explains the interaction among mean annual temperature, annual precipitation, and latitude.

By using the above seven formulae, we tried three statistical models, these being the additive model, additive mixed model including random effects of study site (i.e. random intercept for each site), and additive mixed model including random effects of study site (i.e. random intercept for each site) and random variation for each study site. We also tried a linear mixed model (assuming linear relationship between CT concentration and the parameters), but only the results of the additive models are presented here in the main text because Akaike's Information Criterion (AIC) of the linear models were higher than those of additive models (Table S2). AIC of the additive models with different formulae were compared to select the best model to describe the relationship between mean annual temperature, annual precipitation, and latitude. The additive models were performed by using 'mgcv' package of R (Wood 2004). To test the dependence of CT abundance on methodologies (i.e. extract solution and standard substance for developing calibration curve), we took the residuals of the best model, and examined the effects of the methodologies on the residuals by Kruskal-wallis test. This approach enabled us to test the methodological dependence on CT abundance, excluding the effects of mean annual temperature, annual precipitation, and latitude. All statistical analyses were performed by using a free statistical software $R$ ( $R$ Development Core Team 2009).

\section{RESULTS}

\section{Model Selection}

The formula considering mean annual temperature, annual precipitation, and latitude with random effects of study site and random variation for study site had the lowest AIC among the examined models (Table 1). Generally, AIC decreased in the following order: additive model $>$ additive mixed model with random effects of study site $>$ additive mixed model with random effects of study site and random variation for study site (Table 1). The formulae that considered mean annual temperature, annual precipitation, and latitude were always the best model within a particular statistical model. Based on AIC, CT $\sim \mathrm{s}$ (mean annual temperature $)+s$ (annual precipitation $)+s($ absolute latitude $)$ is the best model to explain CT abundance in our data set.

Table 1. AIC of the Seven Different Model Formulae with Three Additive Models

\begin{tabular}{|c|c|c|c|}
\hline Model structure & $\begin{array}{l}\text { Additive } \\
\text { model }\end{array}$ & $\begin{array}{c}\text { Additive } \\
\text { mixed model } \\
\text { (random } \sim \text { site) }\end{array}$ & $\begin{array}{c}\text { Additive } \\
\text { mixed model } \\
\text { (random } \sim \text { site, } \\
\text { variance } \sim \text { site) }\end{array}$ \\
\hline $\mathrm{CT} \sim \mathrm{s}(\mathrm{MAT})$ & 9532.886 & 9280.392 & 8535.373 \\
\hline $\mathrm{CT} \sim \mathrm{s}($ Precipitation $)$ & 9499.654 & 9282.841 & 8538.768 \\
\hline $\mathrm{CT} \sim \mathrm{s}(\operatorname{absLAT})$ & 9505.803 & 9279.767 & 8537.717 \\
\hline $\begin{array}{l}\mathrm{CT} \sim \mathrm{s}(\mathrm{MAT})+ \\
\mathrm{s}(\text { Precipitation })\end{array}$ & 9434.545 & 9274.267 & 8529.780 \\
\hline $\begin{array}{l}\mathrm{CT} \sim \mathrm{s}(\mathrm{MAT})+ \\
\mathrm{s}(\mathrm{absLAT})\end{array}$ & 9424.985 & 9271.402 & 8528.649 \\
\hline $\begin{array}{c}\mathrm{CT} \sim \mathrm{s}(\text { Precipitation })+ \\
\mathrm{s}(\text { absLAT })\end{array}$ & 9415.849 & 9276.150 & 8533.648 \\
\hline $\begin{array}{c}\mathrm{CT} \sim \mathrm{s}(\mathrm{MAT})+ \\
\mathrm{s}(\text { Precipitation })+ \\
\mathrm{s}(\text { absLAT })\end{array}$ & 9386.213 & 9266.654 & 8523.942 \\
\hline
\end{tabular}

Bold values indicate the lowest AIC within each column. Mean annual temperature, annual precipitation, and absolute latitude are abbreviated as MAT, Precipitation, and absLAT, respectively.

Effects of Climatic Properties, Latitude, and Methodologies on the Foliar CT Abundance

Mean annual temperature significantly and positively affected CT abundance (Figs. 2a and 3a). On the contrary, 

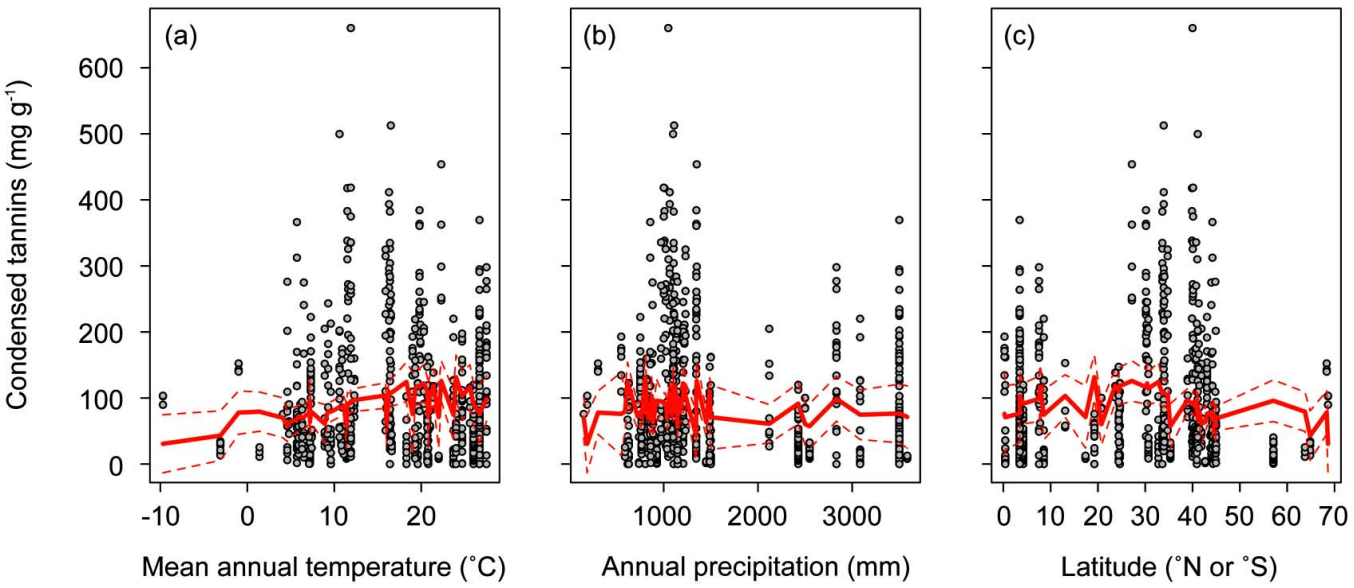

Fig. (2). The relationships between the concentration of CT's versus climatic parameters and latitude. The concentration of CT's versus mean annual temperature (a), annual precipitation (b), and latitude (c). Light gray circles indicate all data points in our data set. Red solid lines indicate the predicted values estimated from the additive mixed model of formula: $\mathrm{CT} \sim \mathrm{s}($ mean annual temperature $)+\mathrm{s}($ annual precipitation $)+s$ (latitude). Red dashed lines indicate $95 \%$ confidential intervals. Random effect and variation are assumed for each study site (see detail in Methods).

annual precipitation and latitude did not have significant effects on CT abundance (Figs. 2b, $\mathbf{c}$ and $\mathbf{3 b}$, c). The variations in CT abundance were large, and different among study sites (Fig. 2). The overall range of foliar CT concentrations was wide, ranging from $0-660 \mathrm{mg} \mathrm{g}^{-1}$ (Fig. 2).

We briefly explored the effects of the methodologies on $\mathrm{CT}$ abundance by examining the residual pattern of the best model. Although previous studies indicated that different methodologies result in different quantification results of $\mathrm{CT}$, we did not find any clear evidence that standard substance nor extract solution affected CT abundance if we had taken the effects of mean annual temperature, annual precipitation, and latitude into account (Fig. S1, Kruskalwallis test, $P>0.05$ ).

\section{DISCUSSION}

Although the data on the CT abundance in tree leaves provides insights into ecosystem processes, such as herbi- vory and decomposition, there have been few investigational studies on CT abundance at the global scale. In this study, we find evidence that mean annual temperature has significant influences on CT abundance at the global scale.

\section{Effects of Climatic Conditions and Latitude on CT Abundance}

Mean annual temperature was significantly and positively correlated with CT values (Figs. 2a and 3a). Some previous studies have also found CT abundance to be higher in a warmer region than in a cooler region (Coley and Barone 1996; Hallam and Read 2006). Our study indicated that this trend could be true even at the global scale. In warmer regions, plants are likely to achieve higher photosynthetic activity than those in colder regions (Farquhar et al. 1980). Thus, the plants may be able to allocate more carbon to carbon-based defensive substances such as CT's. Furthermore, some classic studies have indicated that potential herbivore pressure could be higher in warmer
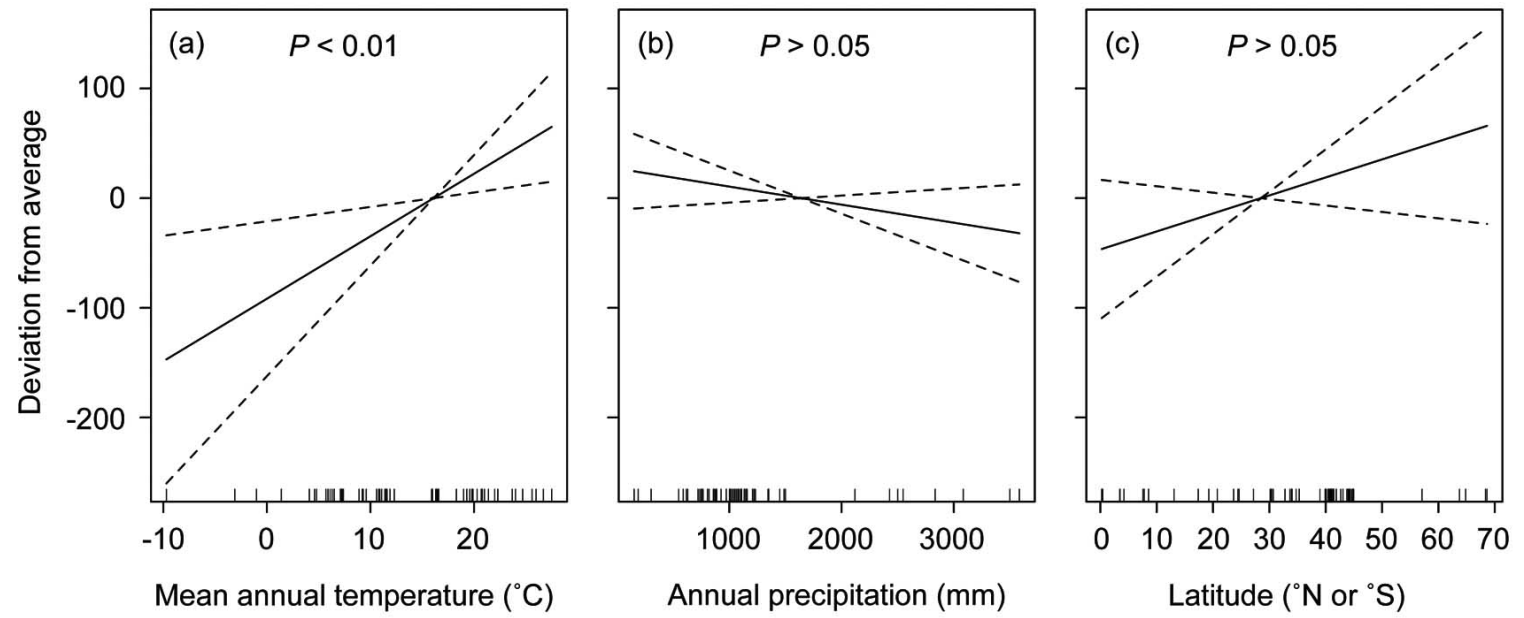

Fig. (3). Estimated effects of climatic parameters on the concentration of CT's. Effects of mean annual temperature (a), annual precipitation (b), and latitude (c) on the concentration of CT's. Solid lines indicate estimated effects of the climatic parameters. Dashed lines indicate $95 \%$ confidential lines. 
regions than in colder regions (Coley and Barone 1996). Therefore, a higher allocation to defensive substances may be advantageous for plants growing in the warmer regions. However, some other studies did not find clear relationships between CT abundance and air temperature or latitude (Stark et al. 2008). Adams et al. (2009a) also showed only limited latitudinal trends in the concentrations of CT's, or other defensive compounds. Therefore, in order to conclude that CT abundance increases precisely with increasing air temperature, more extensive research is needed.

Though we found a significant positive relationship between mean annual temperature and CT abundance, we could still find a very low or zero abundance of foliar CT even in the warmer regions where the potential herbivore pressure can be high. One possible explanation is that plants often have other defensive strategies than direct carbonbased defense such as CT's. For example, tropical plants with a higher intensity of ant-defense (i.e., ant plants) contain a lesser amount of the direct defensive substrate than those with a lower intensity of ant-defense (Itioka 2005). These variations in plant anti-herbivore defense may contribute to our findings that some plant species did not contain foliar CT's, even in the tropical regions. Extract solutions and standard substance did not have significant influence on the quantification of the CT abundance if we had taken the effects of mean annual temperature, annual precipitation, and latitude into account, although previous research found that some methodologies have effects on the quantification of the CT abundance (Cork and Krockenberger 1991).

\section{Unconsidered Factors and Problems in the Quantification of CT's}

Our results showed that $\mathrm{CT}$ abundance is influenced by mean annual temperature, but not by annual precipitation and latitude. However, before concluding that air temperature significantly influences the CT abundance in tree leaves at the global scale, we have to consider some other factors that possibly impact on the CT abundance. The literature on CT's seldom included all tree species occurring within a study site, and the relative abundance of each tree species was also seldom provided. Therefore, it is hard to know the 'real' representative mean $\mathrm{CT}$ concentration in a unit area of the site because the mean value of the site CT's should be provided by calculating CT abundance of each species standardized to the species relative abundance to account for different proportions of the flora in the site (Oates et al. 1990). In addition, we did not test phylogenic effects of plant species on CT abundance, and this might be worth considering as a factor to explain CT abundance.

Other methodologies or sampling designs could also affect the quantification of CT's. Namely, the drying procedure (Cork and Krockenberger 1991), developmental stages of a leaf (Rehill et al. 2006) and nutrient availability (McKey et al. 1978) are also known to influence the concentrations of foliar CT's. Since foliar CT's also affect soil nutrient availability by regulating the soil decomposition process, the interactions between soil nutrient availability and foliar CT's cause important feedback effects (Northup et al. 1998; Ushio et al. 2009). However, these factors could not be considered in this study.
Furthermore, although we discussed that CT's might increase partly due to a higher grazing pressure of herbivores in warmer regions, this idea is now also put in some doubt by some recent studies. For example, Adams et al. (2009b) compared leaf damage area between tropical and temperate regions by using standardized sampling methods and found that there were no distinct differences in the damage area of leaves per unit time between these regions. Another study by the same authors did not find the 'expected' clear latitudinal trend towards more herbivory in warmer climates in North American forests (Adams and Zhang 2009). Thus, comprehensive studies with standardized methods are required to improve understanding of the relationship between herbivory and air temperature or latitude.

\section{Conclusions}

In conclusion, the findings of this study showed that the concentration of CT's in tree leaves was significantly associated with mean annual temperature, a finding that is consistent with the conclusions of older studies and the traditional expectations of theory. However, as some studies have reported previously, the results can be significantly affected by methodologies and other experimental designs, which are not yet standardized. Phylogeny of plant species, which were not included in our analysis, is also important factor to be considered. These factors contribute to the lack of current understanding of the global distribution pattern of CT's in tree leaves, which in turn prevents understanding of ecosystem processes. Comprehensive research with standardized methods will greatly improve our understanding of CT abundance in tree leaves and the mechanisms of ecosystem processes.

\section{ACKNOWLEDGEMENTS}

We greatly thank the authors who provided the climatic data from the study sites. We thank Dr. Hiroyuki Takemoto for his valuable comments on the rough draft of this manuscript. This study was supported in part by Global COE program A06 to Kyoto University. M.U. was supported by Grand-in-Aid for JSPS Fellows (21-1526).

\section{CONFLICT OF INTEREST}

None declared.

\section{SUPPLEMENTARY MATERIAL}

Supplementary material is available on the publishers Web site along with the published article.

\section{REFERENCES}

Adams, J M, Rehill, B, Zhang, Y \& Gower, J (2009a) A test of the latitudinal defense hypothesis: Herbivory, tannins and total phenolics in four North American tree species. Ecological Research, 24, 697-704.

Adams, J M \& Zhang, Y (2009) Is there more insect folivory in warmer temperate climates? A latitudinal comparison of insect folivory in eastern North America. Journal of Ecology, 97, 933-40.

Adams, J M, Zhang, Y, Basri, M \& Shukor, N (2009b) Do tropical forest leaves suffer more insect herbivory? a comparison of tropical 
versus temperate herbivory, estimated from leaf litter. Ecological Research, 24, 1381-92.

Bryant, J P, Chapin III, F S \& Klein, D R (1983) Carbon/nutrient balance of boreal plants in relation to vertebrate herbivory (hare Lepus capensis). Oikos, 40, 357-68.

Coley, P D \& Aide, T M (1991) A comparison of herbivory and plant defenses in temperate and tropical broad-leaved forests, New York, Wiley \& Sons.

Coley, P D \& Barone, J A (1996) Herbivory and plant defenses in tropical forests. Annual Review of Ecology and Systematics, 27, 305-35.

Coley, P D, Bryant, J P \& Chapin, F S (1985) Resource availability and plant antiherbivore defense. Science, 230, 895-99.

Cork, S J \& Krockenberger, a K (1991) Methods and pitfalls of extracting condensed tannins and other phenolics from plants: Insights from investigations on Eucalyptus leaves. Journal of Chemical Ecology, 17, 123-34.

Farquhar, G D, Von Caemmerer, S \& Berry, J A (1980) A biochemical model of photosynthetic $\mathrm{CO} 2$ assimilation in leaves of $\mathrm{C} 3$ species. Planta, 149, 78-90.

Feeny, P (1970) Seasonal changes in oak leaf tannins and nutrients as cause of spring feeding by winter moth caterpillars. Ecology, 51, 565-81.

Forkner, R E, Marquis, R J \& Lill, J T (2004) Feeny revisited: condensed tannins as anti-herbivore defences in leaf-chewing herbivore communities of Quercus. Ecological Entomology, 29, 174-87.

Hagerman, a E \& Butler, L G (1981) The specificity of proanthocyanidinprotein interactions. Journal of Biological Chemistry, 256, 4494-7.

Hagerman, a E, Riedl, K M, Jones, G A, Sovik, K N, Ritchard, N T, Hartzfeld, P W \& Riechel, T L (1998) High molecular weight plant polyphenolics (tannins) as biological antioxidants. Journal of Agricultural and Food Chemistry, 46, 1887-92.

Hallam, A \& Read, J (2006) Do tropical species invest more in antiherbivore defence than temperate species? A test in Eucryphia (Cunoniaceae) in eastern Australia. Journal of Tropical Ecology, 22, 41-51.

Itioka, T (2005) Diversity of Anti-Herbivore Defenses in Macaranga. In: Roubik, D W, Sakai, S \& Karim, A A H (eds.) Pollination Ecology and the Rain Forest. New York, USA: Springer.
Kraus, T E C, Dahlgren, R A \& Zasoski, R J (2003) Tannins in nutrient dynamics of forest ecosystems - A review. Plant and Soil, 256, 4166.

Mckey, D, Waterman, P G, Mbi, C N, Gartlan, J S \& Struhsaker, T T (1978) Phenolic content of vegetation in two African rain forests: Ecological implications. Science, 202, 61-4.

Norris, M \& Oppenheim, C (2007) Comparing alternatives to the Web of Science for coverage of the social sciences' literature. Journal of Informetrics, 1, 161-9.

Northup, R R, Dahlgren, R A \& Mccoll, J G (1998) Polyphenols as regulators of plant-litter-soil interactions in northern California's pygmy forest: A positive feedback? Biogeochemistry, 42, 189-220.

Northup, R R, Yu, Z, Dahlren, R A \& Vogt, K A (1995) Polyphenol control of nitrogen release from pine litter. Nature, 377, 227-9.

Oates, J F, Withesides, H G, Davies, G A, Waterman, P G, Green, M S, Dasilva, L G \& Mole, S (1990) Determinants of variation in tropical forest primate biomass: new evidence from West Africa. Ecology, 71, 328-43.

Porter, L J, Hrstich, L N \& Chan, B G (1986) The conversion of procyanidins and prodelphinidins to cyanidin and delphinidin. Phytochemistry, 25, 223-30.

R Development Core Team (2009) R: A language and environment for statistical computing. In: R foundation for statistical computing (ed.). Vienna, Austria.

Rehill, B J, Whitham, T G, Martinsen, G D, Schweitzer, J A, Bailey, J K \& Lindroth, R L (2006) Developmental trajectories in cottonwood phytochemistry. Journal of Chemical Ecology, 32, 2269-85.

Stark, S, Julkunen-Tiitto, R, Holappa, E, Mikkola, K \& Nikula, A (2008) Concentrations of foliar quercetin in natural populations of white birch (Betula pubescens) increase with latitude. Journal of Chemical Ecology, 34, 1382-91.

Ushio, M, Miki, T \& Kitayama, K (2009) Phenolic control of plant nitrogen acquisition through the inhibition of soil microbial decomposition processes: a plant-microbe competition model. Microbes and Environments, 24, 180-7.

Wood, S N (2004) Stable and efficient multiple smoothing parameter estimation for generalized additive models. Journal of the American Statistical Association, 99, 673-86.

Received: February 01, 2011

Revised: June 06, 2011

Accepted: June 06, 2011

(C) Ushio and Adams; Licensee Bentham Open.

This is an open access article licensed under the terms of the Creative Commons Attribution Non-Commercial License (http:/creativecommons.org/ licenses/by-nc/3.0/), which permits unrestricted, non-commercial use, distribution and reproduction in any medium, provided the work is properly cited. 